\title{
Education in arthroscopy, sports medicine and knee surgery
}

\author{
Anastasios Georgoulis · Pietro Randelli
}

Published online: 1 June 2011

(C) Springer-Verlag 2011

Education in arthroscopy, sports medicine and knee surgery has now reached a very satisfactory level. This can be attributed to (1) the fine staff of medical doctors in general orthopaedics who are interested in working with arthroscopy and sports medicine, (2) the increased level of quality and attractiveness in arthroscopy, sports medicine and knee surgery and (3) innovations in several areas and the evolution of the expertise related to research and publications in peer-reviewed journals. These factors have led to a substantial improvement in the quality of education in arthroscopy, sports medicine and knee surgery. This high education level should be standardised and further improved.

The principal qualifications of a surgeon specialising in our field are as follows. He/she has to be (1) a good surgeon, (2) a good medical doctor, (3) a good team physician and (4) a good researcher. Our efforts should focus on these areas if we want to ensure that our field of interest continues to appeal to young colleagues who want to work hard and make a name for themselves in the orthopaedic community. For this reason, there is a need for good centres that are able to offer work of high quality, fellowships, organised workshops and multi-centre research programmes.

In contrast to the USA, Europe is not as homogeneous in the area of education focusing on arthroscopy, sports

\footnotetext{
A. Georgoulis

Department of Orthopaedic Surgery, Orthopaedic Sports

Medicine Center, University of Ioannina, Ioannina, Greece

e-mail: georgoulis@osmci.gr

P. Randelli $(\bowtie)$

Department Scienze Medico Chirurgiche, IRCCS Policlinico

San Donato, University of Milano, Via Morandi 30,

20097 San Donato Milanese, Milan, Italy

e-mail: pietro.randelli@unimi.it
}

medicine and knee surgery. There are several centres that are famous at national level for their clinics, research, residencies or sub-speciality programmes. There are also universities offering post-graduate programmes in sports medicine or the biomechanics of sport, but the homogenisation of these centres is still lacking.

Workshops in the USA play a central role in education and there are several workshop programmes related to arthroscopy which are incorporated in the programme at the AANA and the AOSSM. In Europe, some very good workshops of high quality are organised and there are some centres like the one at SFA (Société Française d'Arthroscopie), in Strasbourg, France, and the Nicola's Foundation Research and Teaching Centre, in Arezzo, Italy, both of which have the standard equipment and organisation like those in the USA. We need to incorporate all these workshops in a European programme, to give them a central role in education in Europe as well.

Some European countries have a sub-speciality in sports medicine or surgical arthroscopy. We need to establish a trans-European sub-speciality with a compact educational programme, which includes clinical experience, organised workshops, an introduction to scientific writing and reviews of arthroscopy and sports medicine and familiarisation with the biomechanics of sport.

Fellowships in arthroscopy and sports medicine after the residence programme need to be established in Europe as well. They are advertised in the majority of other countries, thereby offering a better mixture of people when it comes to the continuity of good schools of arthroscopy, sports medicine and knee surgery.

Far more attention should focus on education as a team physician. Ideally, a specific educational programme for team physicians, including guidelines for continuing the education of team physicians, should, be established. This 
should not only include education in injury treatment but should also focus far more on prevention, as well as familiarisation with possible fouls during a game that can cause serious injuries to the players, so that the field doctor is able immediately to recognise the severity of these injuries and react accordingly.

In addition, we have to encourage members of our community to introduce sports injuries or sports medicine lessons in the educational programme at their universities, even as an optional lesson. This is a good way to attract young people from university level.

ESSKA has a very important role to play in the homogenisation of education in arthroscopy, sports medicine and knee surgery in Europe. The best way is to establish a structured programme with final examinations, while taking account of the most difficult rules for any European countries that have established something in this direction.

The board of ESSKA is actually involved in developing a close relationship with AOSSM regarding the organisation of sub-speciality certification in the field. In the next 4-6 years, it will be possible to draw up a curriculum in arthroscopy, sports medicine and knee surgery, with a final examination.

ESSKA's commitment to education has grown in the last few years. The biennial meeting represents a unique to obtain an update on arthroscopy, sports medicine and knee surgery. The Oslo meeting, held in June 2010, attracted a huge number of participants, comprising around 2,600 orthopaedic doctors from all over the world. ESSKA's members received, at no cost, 5 books on knee, shoulder and ankle surgery, plus 4 DVDs. This educational effort was made possible by hard work involving authors from the entire ESSKA committee. The next biennial meeting will be in Geneva, Switzerland, on 2-5 May 2012, at which the "state of the art" will be presented in several parallel sessions.
It is also interesting to note that the backbone of ESSKA is the fellowship programme that includes the worldfamous international travel fellowships in conjunction with the AOSSM, APOSSM and SLARD. The best young European orthopaedic doctors can travel, with famous godfathers, to the most important sports medicine centres in North America, the Pacific countries and South America respectively. These fellowships are sponsored by an educational grant from DJO (Don Joy Orthopaedics) and Smith and Nephew.

The fellowship programme also includes some European visiting fellowships in arthroscopy, which enable applicants to visit the best ESSKA teaching centres. These fellowships are sponsored by an educational grant from Arthrex and Smith and Nephew.

Finally, ESSKA's education programme includes two total knee replacement fellowships. Educational grants from Tornier and Zimmer enable several colleagues to learn the most advanced techniques at selected European ESSKA teaching centres.

In the last few months, the board of ESSKA has been making a real effort to reinforce these important programmes still further, by organising a "senior surgeon" visiting fellowship in Europe. This fellowship will allow more mature surgeons to improve their skills with a 1-week fellowship at selected centres.

All these fellowships are listed on the website at: www.esska.org.

In conclusion, education in arthroscopy, sports medicine and knee surgery has improved in Europe in the past few years, in spite of different rules and organisations in the various countries and at their universities. ESSKA and the national scientific communities have a key role to play in education, with the common final aim of developing European certification arthroscopy, sports medicine and knee surgery. 